\title{
Evaluación del comportamiento electroquímico del extracto de nopal (Opuntia Ficus-Indica) como posible inhibidor de corrosión
}

\author{
Araceli Mandujano-Ruíz ${ }^{\mathrm{a}, 凶}$, Jorge Morales-Hernández ${ }^{\mathrm{a}}$, Héctor Herrera-Hernández ${ }^{\mathrm{b}}$, Luis E. Corona- \\ Almazán ${ }^{\mathrm{b}}$, José M. Juárez García ${ }^{\mathrm{c}}$ \\ ${ }^{a}$ Centro de Investigación y Desarrollo Tecnológico en Electroquímica (CIDETEQ), Parque Tecnológico Querétaro s/n, Sanfandila, \\ Pedro Escobedo, C.P. 76703, Querétaro, México \\ ${ }^{\mathrm{b}}$ Universidad Autónoma del Estado de México, IIN-Área de Electroquímica Aplicada y Corrosión de Materiales, Blvd. \\ Universitario S/N, Predio San Javier, Atizapán de Zaragoza, C.P. 54500, Estado de México, México \\ c Centro Nacional de Metrología, Metrología de Materiales, km 4.5 Carretera a Los Cués, El Márques Querétaro, C.P. 76246, México \\ $\triangle$ Autor para la correspondencia: amandujano@cideteq.mx
}

RESUMEN: La corrosión es uno de los principales problemas del deterioro en componentes, herramentales, equipos e inclusive a nivel estructural, ejemplo de esto son los aceros al carbono. En el presente trabajo se estudió la capacidad de inhibición a la corrosión de un extracto orgánico biodegradable proveniente de la planta del Nopal (Opuntia ficus-indica) para la protección del acero al carbono tipo AISI 1018, al agregar el 50\% v/v del extracto de Nopal (EN) en una solución de $\mathrm{H}_{2} \mathrm{SO}_{4}\left(0,6\right.$ mol.1 $\left.{ }^{-1}\right)$. Se utilizaron las técnicas de Resistencia a la Polarización (LPR) y Espectroscopía de Impedancia Electroquímica (EIS) para determinar la eficiencia de inhibición (IE) a temperatura ambiente durante $24 \mathrm{~h}$ y obtener las velocidades de corrosión ( $\mathrm{V}_{\text {corr }}$ ), posteriormente se llevó a cabo la evaluación metalográfica para registrar el daño superficial. Los resultados mostraron una reducción de la $\mathrm{V}_{\text {corr }}$ con una IE máxima del 84\% al adicionar el inhibidor orgánico EN.

PALABRAS CLAVE: Espectroscopía de impedancia electroquímica; Extracto de nopal; Inhibidor de corrosión; Resistencia a la polarización

Citar como/Citation: Mandujano-Ruíz, A.; Morales-Hernández, J.; Herrera-Hernández, H.; Corona-Almazán, L.E.; Juárez García, J.M. (2017) "Evaluación del comportamiento electroquímico del extracto de nopal (Opuntia FicusIndica) como posible inhibidor de corrosión”. Rev. Metal. 53(4):e108. http://dx.doi.org/10.3989/revmetalm.108

\begin{abstract}
Evaluation of Electrochemical Behavior of Nopal Extract (Opuntia Ficus- Indica) as Possible Corrosion Inhibitor. Corrosion is one of the main problems of degradation in components, tooling, equipment and even in structural applications, examples of this are the carbon steels. In the present work, the capacity of corrosion inhibition of a biodegradable organic extract from the Nopal plant (Opuntia ficus-indica), for the protection of carbon steel type AISI 1018 was studied adding 50\% v/v of the Nopal extract (EN) in a solution of $\mathrm{H}_{2} \mathrm{SO}_{4}\left(0.6 \mathrm{~mol}_{1} \mathrm{I}^{-1}\right)$. Polarization Resistance (LPR) and Electrochemical Impedance Spectroscopy (EIS) techniques were used for the electrochemical evaluation at room temperature for $24 \mathrm{~h}$ in order to obtain corrosion rates $\left(\mathrm{V}_{\text {corr }}\right)$ and inhibition efficiency (IE). Metallographic examination was also carried out to register the surface damage by corrosion. The results showed a reduction of the $\mathrm{V}_{\text {corr }}$ with a maximum IE value of about $84 \%$ by adding the organic- liquid extracted from Nopal.
\end{abstract}

KEYWORDS: Corrosion inhibitor; Electrochemical impedance spectroscopy; Nopal extract; Polarization resistance

ORCID ID: Araceli Mandujano-Ruíz (http://orcid.org/0000-0003-2074-1116); Jorge Morales-Hernández (http://orcid. org/0000-0002-8892-128X); Héctor Herrera-Hernández (http://orcid.org/0000-0002-1485-3624); Luis E. CoronaAlmazán (http://orcid.org/0000-0003-1398-9404); José M. Juárez García (http://orcid.org/0000-0002-2303-5420)

Copyright: (C 2017 CSIC. Este es un artículo de acceso abierto distribuido bajo los términos de la licencia Creative Commons Attribution (CC BY) España 3.0. 


\section{INTRODUCCIÓN}

Los problemas por corrosión en la industria se ven reflejados en pérdidas económicas debido a la degradación de componentes de acero, parada de las unidades de producción por mantenimiento y en un caso extremo, paros por accidentes del personal. Estas problemáticas motivan a diversas ramas de la ciencia e ingeniería a dirigir sus esfuerzos para encontrar alternativas que ayuden a minimizar la presencia de corrosión, la cual también está presente durante el traslado o almacenamiento de las piezas de acero que después de su manufactura presentan una superficie muy activa, siendo susceptibles de presentar corrosión en función del tiempo de almacenamiento y/o traslado.

Algunas prácticas para el traslado y almacenamiento de piezas de acero hacen uso de inhibidores de corrosión a base de aceites industriales donde las piezas son impregnadas o sumergidas evitando que se corroan; sin embargo, al utilizar este método las piezas requieren un postratamiento por lo que tienen que ser lavadas antes de su disposición, agregando así una operación de lavado al proceso para eliminar el inhibidor significando gastos de operación no programados. Otras opciones involucran el uso de papel encerado lo que evita la operación de remoción del inhibidor industrial, sin embargo, el costo es alto y poco eficiente.

En la literatura se han reportado diversos compuestos orgánicos con grupos funcionales que contienen elementos del grupos V y VI tales como: nitrógeno, fosforo, arsénico, azufre, oxígeno y selenio han sido investigados por mostrar un buen desempeño como inhibidor en aceros de bajo carbono, sin embargo, muchos de estos inhibidores no solo son costosos sino que también son peligrosos para el medio ambiente, por lo que su uso se ha reducido debido a las modificaciones en la normatividad ISO 14001, que hace referencia a la restricción en el uso de inhibidores de corrosión sintéticos. De éste modo nace la necesidad de crear formulaciones totalmente amigables con el ambiente, por lo que en los últimos años surge la propuesta del uso de inhibidores verdes "Green inhibitors" generando una nueva línea de investigación de gran impacto tecnológico por su bajo riesgo de contaminación al medio ambiente (Singh y Bockris, 1996; Ismail et al., 2015).

Por lo tanto, el desarrollo de inhibidores orgánicos biodegradables a partir de extractos de plantas ofrece la posibilidad de proteger las piezas de acero para su traslado o almacenamiento temporal sin la necesidad de un proceso de lavado previo a la disposición de éstas y sin la generación de residuos industriales.

La eficiencia de un compuesto orgánico como inhibidor de corrosión depende principalmente de la habilidad de anclarse por interacciones químicas o físicas sobre la superficie del metal formando multicapas de moléculas orgánicas adsorbidas que bloquean los sitios activos de corrosión y retardando las reacciones anódicas y/o catódicas. La estabilidad de dicha capa adsorbida sobre la superficie depende de las propiedades físico-química de las moléculas relacionadas con los grupos funcionales del inhibidor: anillos aromáticos, posible efecto de histéresis, densidad electrónica de átomos donadores, además de otros factores como el ambiente corrosivo y la naturaleza de la interacción entre el orbital $\pi$ de los inhibidores y el orbital $d$ del hierro (Sanyal, 1981; Khaled, 2010; Abreu-Quijano et al., 2011).

Algunos extractos de plantas y frutos que han sido estudiados como inhibidores verdes son: Brungmasia Suaveolens, Cassia Roxburghii, Floripondio, Musa Paradisiaca, Cascara de papa, Aloe Vera y Hojas de Higuera, han reportado un buen comportamiento de inhibición frente a medios ácidos como $\mathrm{HCl}$ ya que han incrementado la resistencia a la corrosión de aceros de bajo carbono utilizando pequeñas concentraciones de estos inhibidores, sin embargo, al incrementar la temperatura su efecto tiende a disminuir (Gopiraman et al., 2011; Ibrahim y Zour, 2011; Herrera-Hernández et al., 2015).

Por otro lado, el Nopal (Opuntia Ficus-Indica) es un vegetal tradicional en México con un alto contenido de fibra; es una planta silvestre que sobrevive en regiones desérticas y que no requiere de mucha agua para su cultivo. Se dice que tiene un desempeño ecológico importante, ya que detiene la degradación del suelo deforestado convirtiendo la tierra árida en productiva. Existen cerca de 1600 especies en 122 géneros de la familia de las cactáceas, de la cual proviene el nopal. Tiene frutos que son comestibles y se conocen con el nombre de tunas. La fibra soluble del Nopal conocida como mucilago, tiene la capacidad de retener hasta 30 veces su peso en agua por lo que ha sido de interés como inhibición de la corrosión en acero de bajo carbono como lo reportan algunos autores (Torres-Acosta et al., 2012; Flores de los Ríos et al., 2015).

\section{MATERIALES Y MÉTODOS}

\subsection{Preparación de los especímenes}

Una barra de acero AISI 1018 se seccionó en especímenes de $25 \mathrm{~mm}$ de diámetros por $5,0 \mathrm{~mm}$ de espesor, éste acero se utilizó como sustrato y electrodo de trabajo (WE, "por sus siglas en inglés") para las pruebas electroquímicas; composición se presenta en la Tabla 1. Cada probeta se sometió a un desbaste con papel de $\mathrm{SiC}$ pasando por los grados del número $80,240,320,400,600$ y 1500 ; posteriormente las muestras se lavaron con agua destilada y se desengrasaron con acetona con un secado final con aire caliente. 


\subsection{Preparación del extracto de nopal (EN)}

Para la preparación del extracto de Nopal se seleccionaron pencas tiernas que se limpiaron (remoción de espinas), cortaron y se desinfectaron con $2 \mathrm{ml} \mathrm{de} \mathrm{Cl}_{2}+1 \mathrm{ml}$ de $\mathrm{Cu}_{2} \mathrm{SO}_{4}$ para garantizar la eliminación de microorganismo y hongos que degraden al extracto. Posteriormente se llevó a trituración en un extractor donde la pulpa resultante se calentó para su maceración con una relación en volumen de agua destilada 2:1 durante una hora a $78^{\circ} \mathrm{C}$. El concentrado líquido se filtró en un tamiz fino para asegurar la separación de los sólidos superiores a $180 \mu \mathrm{m}$, obteniendo el producto final para las pruebas como se observa en la Fig. 1.

\subsection{Sistema electroquímico}

La caracterización electroquímica se llevó a cabo en una celda de acrílico de diseño propio ( GarfiasGarcía et al., 2010; Herrera-Hernández et al., 2015), la cual se ensamblo con un arreglo de 3 electrodos: el acero 1018 responde como electrodo de trabajo (WE), una rejilla de platino como contraelectrodo (CE) y un electrodo de calomelano como electrodo de referencia (RE, "por sus siglas en inglés). Se utilizó una solución de $\mathrm{H}_{2} \mathrm{SO}_{4}\left(0,6 \mathrm{~mol} \cdot \mathrm{l}^{-1}\right)$ como electrolito de prueba preparada con reactivos grado analítico y agua desionizada. Todos los experimentos fueron realizados en condiciones estándar de temperatura y presión. La Fig. 2 ilustra la configuración de la celda electroquímica utilizada.

TABLA 1. Composición del acero SAE 1018

\begin{tabular}{lc}
\hline Elemento & Composición $(\%$ peso) \\
\hline $\mathrm{C}$ & $0,15-0,20$ \\
$\mathrm{Mn}$ & $0,60-0,90$ \\
$\mathrm{P}$ & 0,03 Máx. \\
$\mathrm{S}$ & 0,05 Máx. \\
$\mathrm{Fe}$ & Balance \\
\hline
\end{tabular}

Las pruebas electroquímicas se llevaron a cabo con un potenciostato Bio-Logic ${ }^{\circledR}$ ajustando la secuencia que se ilustra en la Fig. 3. En primer lugar se programó el monitoreo del Potencial a Circuito Abierto (OCP) para asegurar la estabilidad del sistema durante $1 \mathrm{~h}$, seguido de la técnica de Espectroscopía Impedancia Electroquímica (EIS), aplicando un barrido de frecuencias de $100 \mathrm{kHz}$ hasta $10 \mathrm{mHz}$ con $10 \mathrm{mV}$ de amplitud; posteriormente se ejecutó nuevamente la medición de OCP durante $1 \mathrm{~h}$ para acabar la secuencia realizando una Resistencia a la Polarización (LPR) con un rango de potencial de $\pm 25 \mathrm{mV}$ y una velocidad de barrido de $1 \mathrm{mV} \cdot \mathrm{s}^{-1}$; el ciclo OCP-EIS-OCP-LPR se repitió durante $24 \mathrm{~h}$ para registrar el comportamiento final mediante una Curva de Polarización (CP) aplicando un sobrepotencial de $\pm 1 \mathrm{~V}$ a una velocidad de barrido de $1 \mathrm{mV} \cdot \mathrm{s}^{-1}$. Al finalizar la experimentación se realizaron las determinaciones de la velocidad de corrosión del acero 1018 en $\mathrm{H}_{2} \mathrm{SO}_{4}\left(0,6 \mathrm{~mol} \cdot \mathrm{l}^{-1}\right)$ como blanco de referencia y posteriormente se realizaron pruebas con la adición del 50\% v/v del Extracto de Nopal (EN) en el medio corrosivo.

\section{RESULTADOS Y DISCUSIÓN}

El monitoreo del potencial de corrosión ( $\left.\mathrm{E}_{\text {corr }}\right)$ para el comportamiento del acero 1018 en $\mathrm{H}_{2} \mathrm{SO}_{4}$ y con EN quedó registrado en la Fig. 4, en donde se observa que el potencial de corrosión disminuye en función del tiempo con la presencia del extracto de nopal en la solución ácida, recorriéndose a hacia valores más negativos con respecto a la muestra metálica sin inhibidor (blanco), lo que está relacionado con una mayor actividad en la superficie del sustrato.

La técnica LP fue realizada durante las $24 \mathrm{~h}$ de inmersión en ambos medios (blanco y $\mathrm{H}_{2} \mathrm{SO}_{4}+$ EN) que se muestran en la Fig. 5, donde se puede observar que las curvas correspondientes a $\mathrm{H}_{2} \mathrm{SO}_{4}$ + EN se desplazan hacia valores menores tanto en potencial y corriente con respecto al tiempo, lo cual indica un comportamiento de inhibición del tipo catódico. El valor promedio de las curvas con y sin
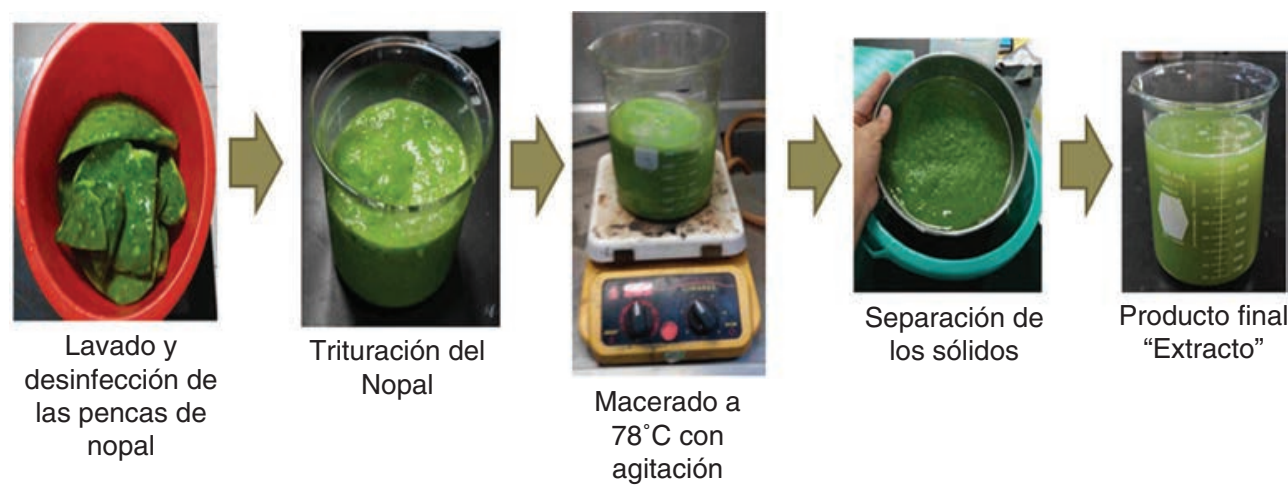

Figura 1. Proceso de Extracción del concentrado de Nopal. 
inhibidor se muestra en la Fig. 6 donde se observa una tendencia a acercarse ambos potenciales, con una disminución en la corriente con la presencia del inhibidor con respecto al blanco; de tal manera que $\mathrm{E}_{\text {corr }}$ de la mezcla $\mathrm{H}_{2} \mathrm{SO}_{4}+\mathrm{EN} \approx \mathrm{E}_{\text {corr }}$ del blanco e $\mathrm{i}_{\text {corr }}$ de $\mathrm{H}_{2} \mathrm{SO}_{4}+\mathrm{EN}<\mathrm{i}_{\text {corr }}$ del blanco, por lo que el comportamiento promedio de inhibición finalmente fue del tipo mixto, sin ningún cambio apreciable en ambas ramas de la curva.

Mediante la extrapolación de Tafel se obtuvieron los valores de las pendientes anódicas $\left(\beta_{\mathrm{a}}\right)$ y catódicas $\left(\beta_{\mathrm{b}}\right)$ para el cálculo del coeficiente de Stern Geary (B), el cruce de las pendientes permitió determinar el valor $E_{\text {corr }}$ e $i_{\text {corr }}$, siendo este último valor dividido entre el área expuesta de la muestra la cual fue de $1 \mathrm{~cm}^{2}$ para obtener una densidad de corriente

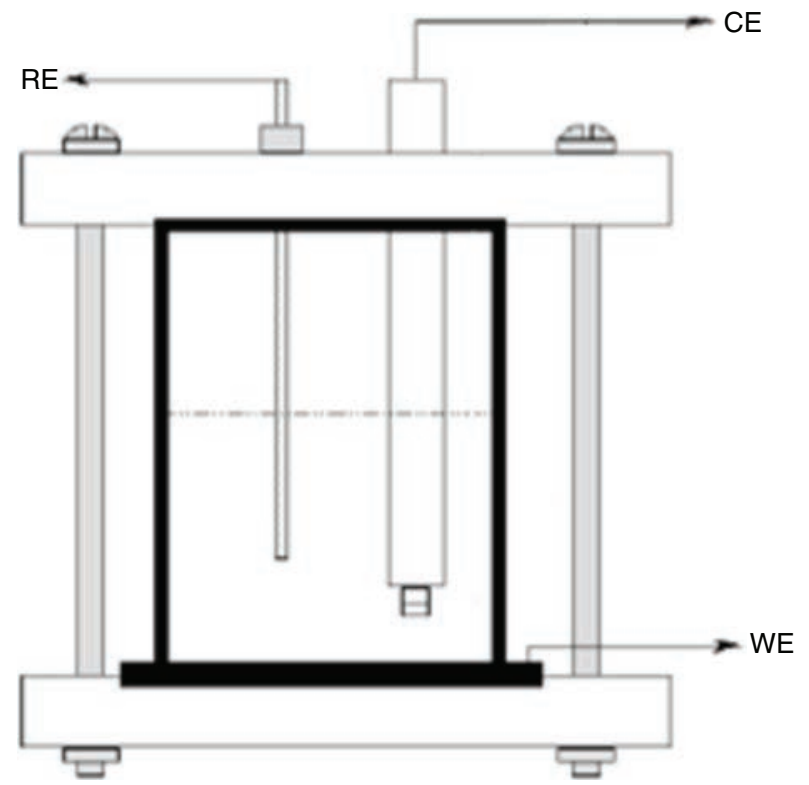

Figura 2. Celda Electroquímica para pruebas de corrosión.
$\mathrm{j}_{\mathrm{o}}\left(\mathrm{A} \cdot \mathrm{cm}^{-2}\right)$ para el cálculo de la resistencia a la transferencia de carga $\left(\mathrm{R}_{\mathrm{p}}\right)$ para la técnica LP por medio de la relación (Ec. (1)):

$$
j_{o}=\frac{B}{R_{P}}
$$

Conociendo $\mathrm{j}_{\mathrm{o}}$ es posible conocer $\mathrm{V}_{\text {corr }}$, por lo tanto, con las modificaciones adecuadas la ecuación de Faraday ahora queda representada por la ecuación siguiente (Ec. (2)):

$$
V_{\text {corr }}=\frac{j_{o} \cdot M}{n \cdot F}
$$

siendo M la masa del metal, $\mathrm{n}$ el número de electrones transferidos, y $F$ la constante de Faraday equivalente a 96500 culombios $/ \mathrm{mol}$.

En la Fig. 7 se presenta el comportamiento de $\mathrm{V}_{\text {corr }}$ obtenidos con ambas técnicas (LPR y EIS) a lo largo de $24 \mathrm{~h}$ de exposición; se puede observar que hay un efecto importante en la disminución de $\mathrm{V}_{\text {corr }}$ con la adición del $50 \%$ v/v de extracto de nopal; disminuyendo la $i_{\text {corr }}$ en un orden de magnitud acorde a los datos reportados en la Tabla 2, donde los valores de $R_{p}$ y $R_{c t}$ obtenidos por ambas técnicas (LPR y EIS), mostraron la misma tendencia a incrementar con la presencia del extracto de nopal (EN) en el medio ácido. Los valores reportados en la Tabla 2, representan un promedio de todas las mediciones obtenidas a lo largo de las $24 \mathrm{~h}$ de exposición.

La técnica de EIS permite separar las contribuciones de resistencia a la transferencia de carga del sistema $\left(R_{c t}\right)$ y efecto de caídas óhmicas debida a la concentración de la solución, es decir, la resistencia de la solución $\left(\mathrm{R}_{\mathrm{sol}}\right)$, este último valor resulta ser bajo $\approx 10 \Omega$, debido a que la concentración del $\mathrm{H}_{2} \mathrm{SO}_{4}$ es alta por lo que al comparar los valores de $\mathrm{R}_{\mathrm{ct}}$ con

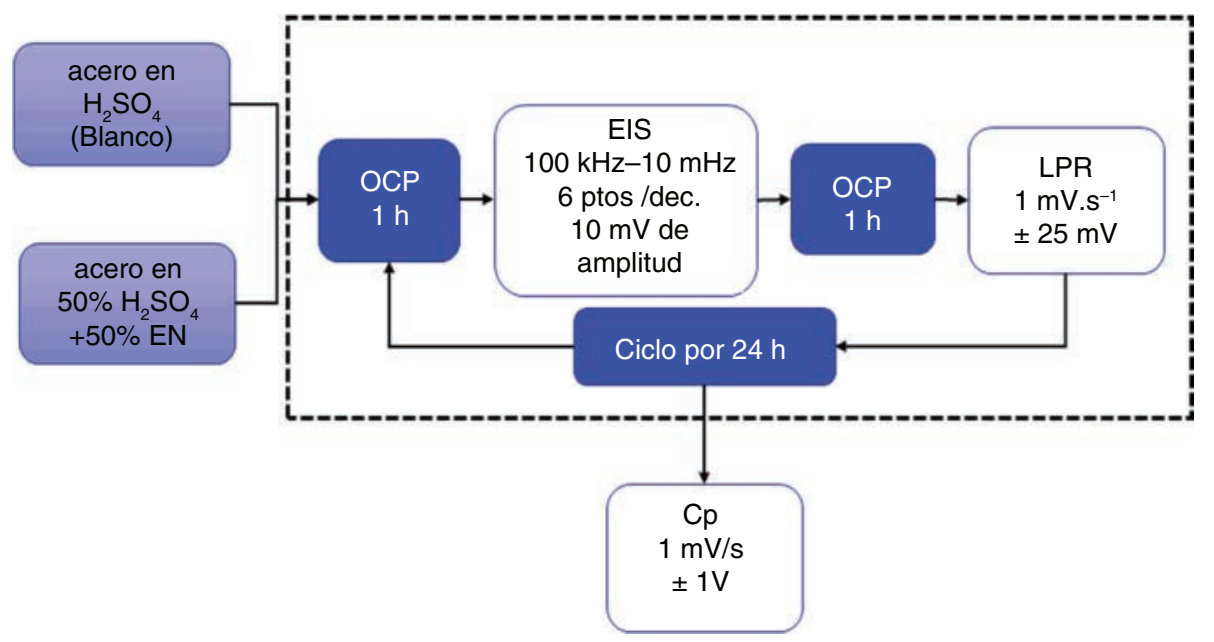

FIgURA 3. Secuencia Experimental. 


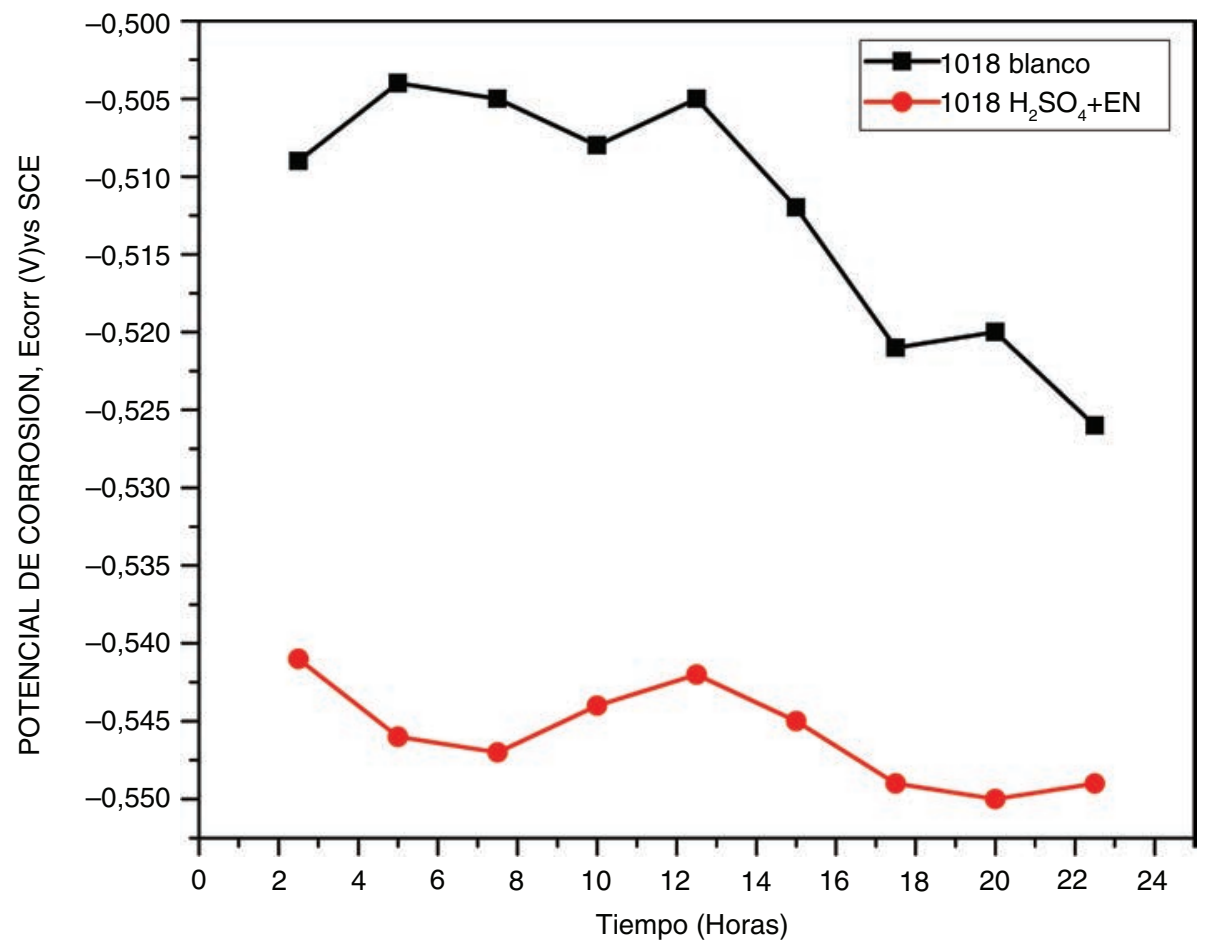

Figura 4. Potencial de Corrosión del acero 1018 con y sin inhibidor.

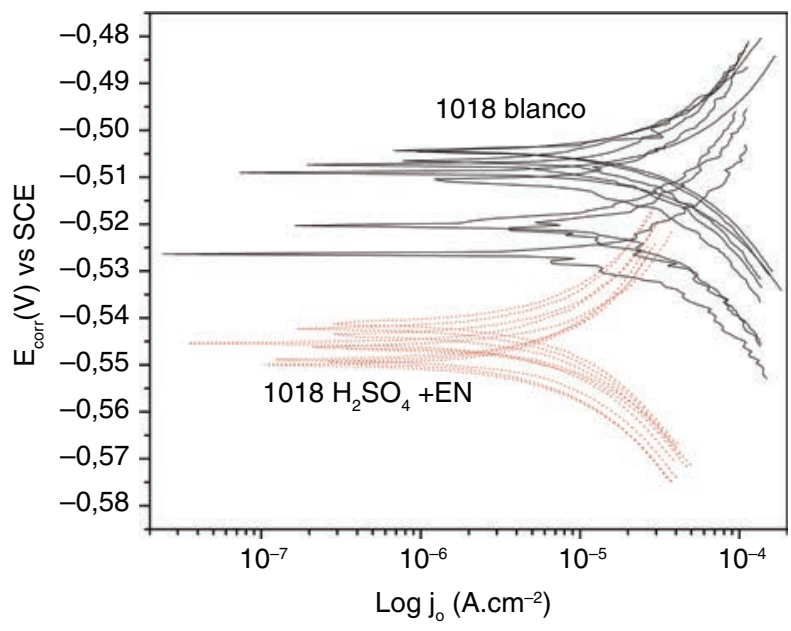

Figura 5. Polarización Lineal en función del tiempo a $\pm 25 \mathrm{mV}$ vs SCE, para el acero 1018 con y sin inhibidor.

respecto a los de $\mathrm{R}_{\mathrm{P}}$ resultan menores para ambos casos (blanco y $\mathrm{H}_{2} \mathrm{SO}_{4}+\mathrm{EN}$ ). También se observó una disminución en el valor de la capacitancia $\left(\mathrm{C}_{\mathrm{dl}}\right)$ lo cual está ligado a una disminución de la constante dieléctrica en las regiones locales en la fase ferrita debido a un mecanismo de adsorción molecular del inhibidor en la interface metal/electrolito, incrementando el espesor de la doble capa electroquímica que está asociado a la capacidad de inhibición de las moléculas del nopal.

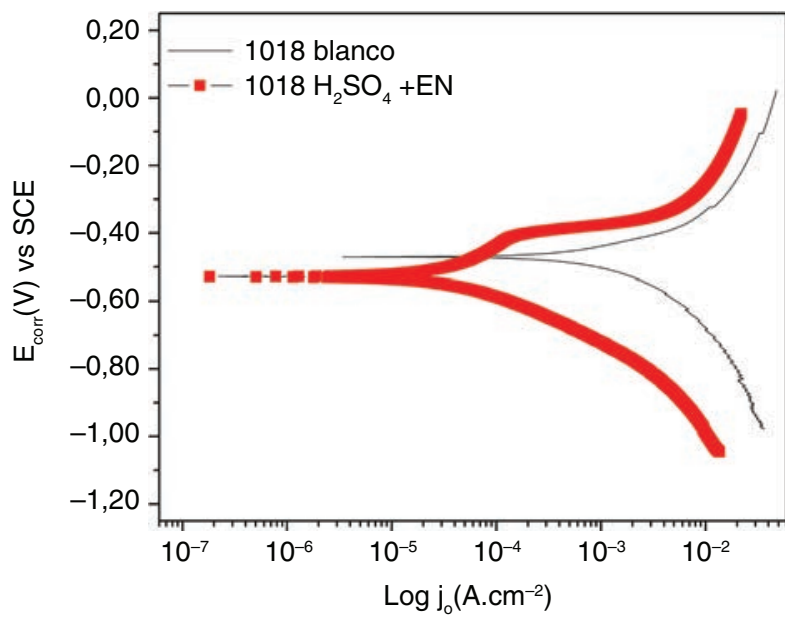

Figura 6. Curvas de Polarización para el acero 1018 con y sin inhibidor.

La Fig. 8 muestra los resultados de EIS, representados mediante los gráficos de Nyquist, en ellos se puede observar el comportamiento del sustrato al adicionar el extracto de Nopal. Se puede observar un incremento en la amplitud del bucle (semicírculo) cuyo valor en el rango de altas frecuencias fue extrapolado mediante un ajuste de la curvatura del semicírculo hasta cruzar con el eje X obteniendo así el valor de $\mathrm{R}_{\mathrm{ct}}$, dichos valores incrementan considerablemente con respecto al blanco. 


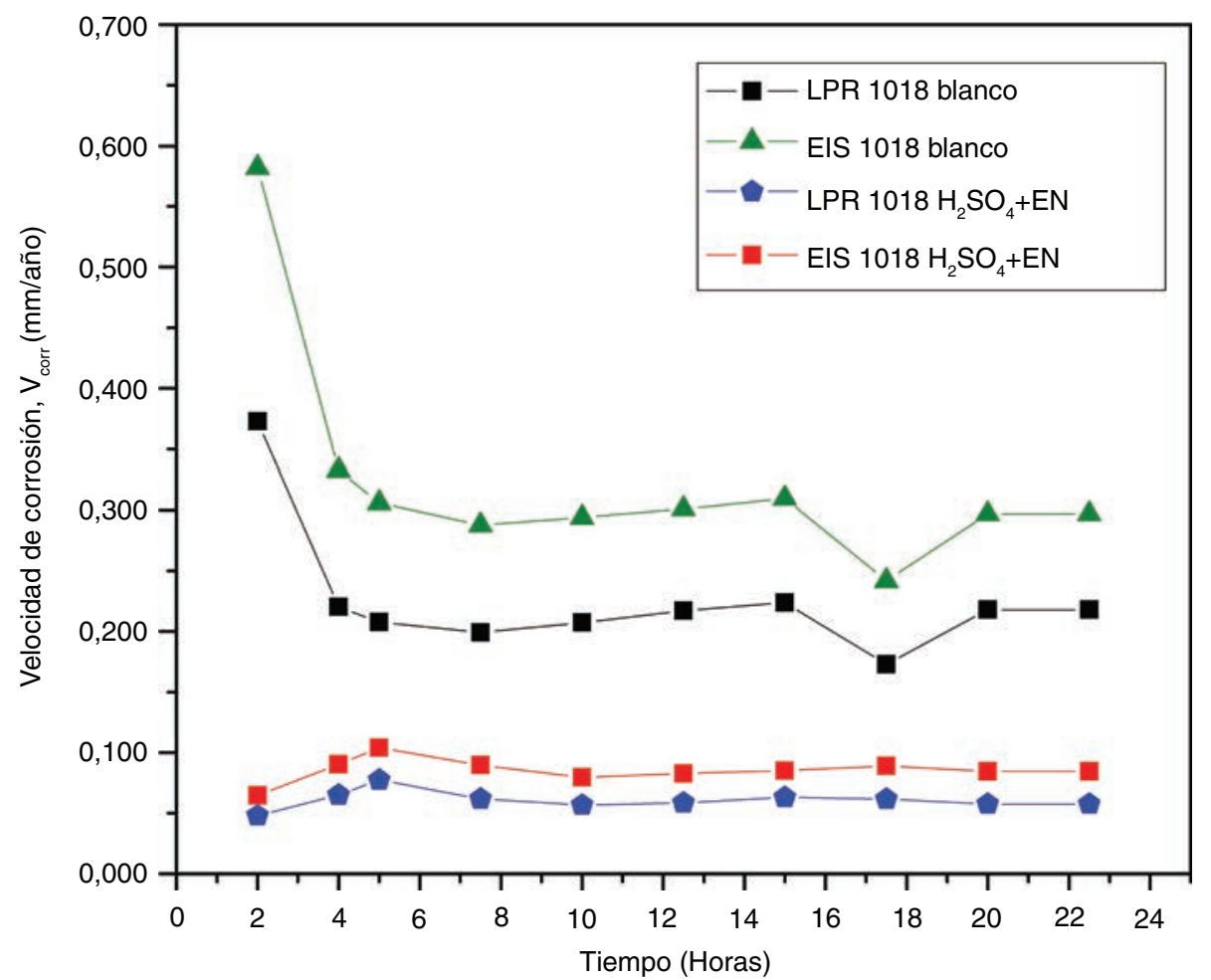

FIGURA 7. Gráfico de velocidad de corrosión vs tiempo, obtenidos mediante los resultados de LPR y EIS.

TABLA 2. Resultados de las técnicas electroquímicas para evaluar al acero 1018 con y sin inhibidor

\begin{tabular}{lcccccc}
\hline & \multicolumn{3}{c}{ LPR } & & \multicolumn{2}{c}{ EIS } \\
\cline { 2 - 4 } \cline { 6 - 7 } Acero $\mathbf{1 0 1 8}$ & $\boldsymbol{j}_{\boldsymbol{o}}\left(\mathbf{A} \cdot \mathbf{c m}^{-2}\right)$ & $\mathrm{E}_{\mathbf{c o r r}}(\mathbf{V}) \mathbf{v s} \mathbf{S C E}$ & $\mathrm{R}_{\mathbf{p}}\left(\mathbf{\Omega} \cdot \mathbf{c m}^{-2}\right)$ & & $\mathrm{R}_{\mathbf{c t}}\left(\mathbf{\Omega} \cdot \mathbf{c m}^{-2}\right)$ & $\mathrm{C}_{\mathrm{dl}}\left(\boldsymbol{\mu} \mathbf{F} \cdot \mathbf{c m}^{-2}\right)$ \\
\hline $\mathrm{H}_{2} \mathrm{SO}_{4}$ & $2,927 \mathrm{E}-05$ & $-0,511$ & 268,182 & & 187,538 & 189 \\
$50 \% \mathrm{H}_{2} \mathrm{SO}_{4}+50 \% \mathrm{EN}$ & $7,856 \mathrm{E}-06$ & $-0,545$ & 1015,013 & & 717,162 & 154 \\
\hline
\end{tabular}

El ajuste del semicírculo, se realizó para cada uno de los ciclos de impedancia tomando como referencia un circuito eléctrico equivalente (CEE) como el que se muestra en la Fig. 9 en la parte superior del gráfico. Debido a la forma del semicírculo de Nyquist se tomó la configuración básica denominada CEE del modelo de Randles, el cual está conformado por una resistencia en serie (resistencia de la solución, $\mathrm{R}_{\mathrm{s}}$ ), acoplado a un elemento de fase constante (CPE), que está en paralelo con otra resistencia atribuida a la interfase metal/electrolito, es decir, $\mathrm{R}_{\mathrm{ct}}$. Conforme se incrementó el tiempo de exposición, el semicírculo muestra una tendencia disminuir ligeramente lo que se puede correlacionar con la alteración de la película de protección formada por el inhibidor en función del tiempo de exposición, al finalizar el monitoreo, los valores de $\mathrm{R}_{\mathrm{ct}}$ de la película de inhibidor no se acercan a los valores de $\mathrm{R}_{\mathrm{ct}}$ del blanco.

La propuesta del CEE se sustenta en la observación de una sola constante de tiempo formada en los diagramas de bode-fase (Fig. 10), donde se puede apreciar un ángulo máximo $\left(\omega_{\theta \max }\right.$ de $60^{\circ}$ para el medio $\mathrm{H}_{2} \mathrm{SO}_{4}+\mathrm{EN}$, mismo que puede estar relacionado con una mejor distribución del compuesto orgánico sobre la superficie del acero, denotando una mejor estabilidad de la capa formada por el inhibidor y que correspondiente con un incremento en la impedancia observado en el gráfico de Bode-Fase.

Para obtener los valores de capacitancia $\left(\mathrm{C}_{\mathrm{dl}}\right)$ a partir del elemento de fase constante (CPE), fue necesaria la obtención de la frecuencia máxima del semicírculo de Nyquist $\left(\omega_{\theta \max }\right)$ así como el exponente a determinado durante el ajuste de CPE, ya que $\mathrm{CPE}=\mathrm{F} \cdot \mathrm{s}^{(\mathrm{a}-1)}: \mathrm{F}$ (Faradios) y s (segundos) (PalomarPardavé et al., 2012). El análisis dimensional arroja valores en " $F$ " pero en la Tabla 2 se presentan en $\mu \mathrm{F}$ y divididos por el área de exposición $\mu \mathrm{F} \cdot \mathrm{cm}^{-2}$. La expresión 3 muestra el cálculo de $\mathrm{C}_{\mathrm{dl}}$ :

$$
C_{d l}=C P E * \omega_{\theta \max }^{(a-1)}
$$




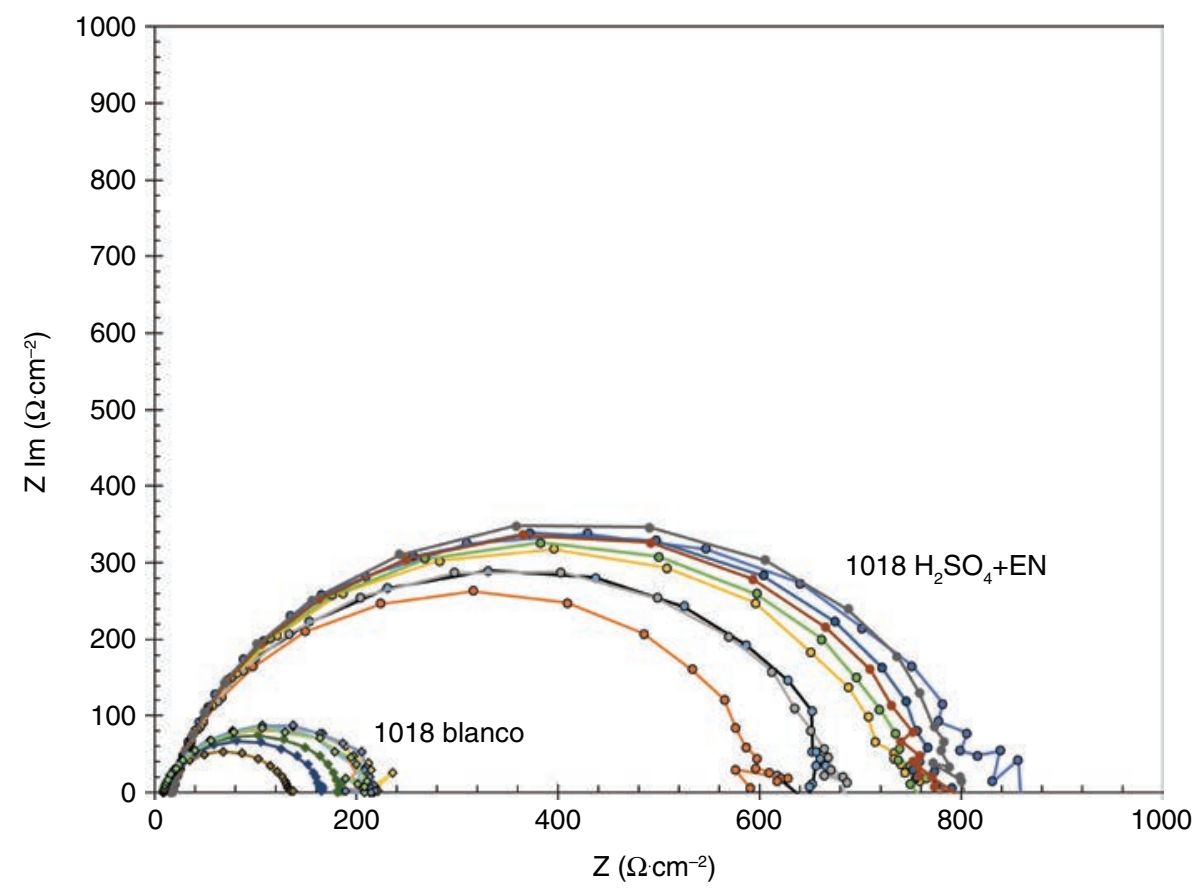

FIgURA 8. Gráficos de Nyquist correspondientes al comportamiento del acero AISI-1018 en $\mathrm{H}_{2} \mathrm{SO}_{4}$ y con la mezcla $50 \% \mathrm{H}_{2} \mathrm{SO}_{4}+$ $50 \%$ EN en función del tiempo de exposición.

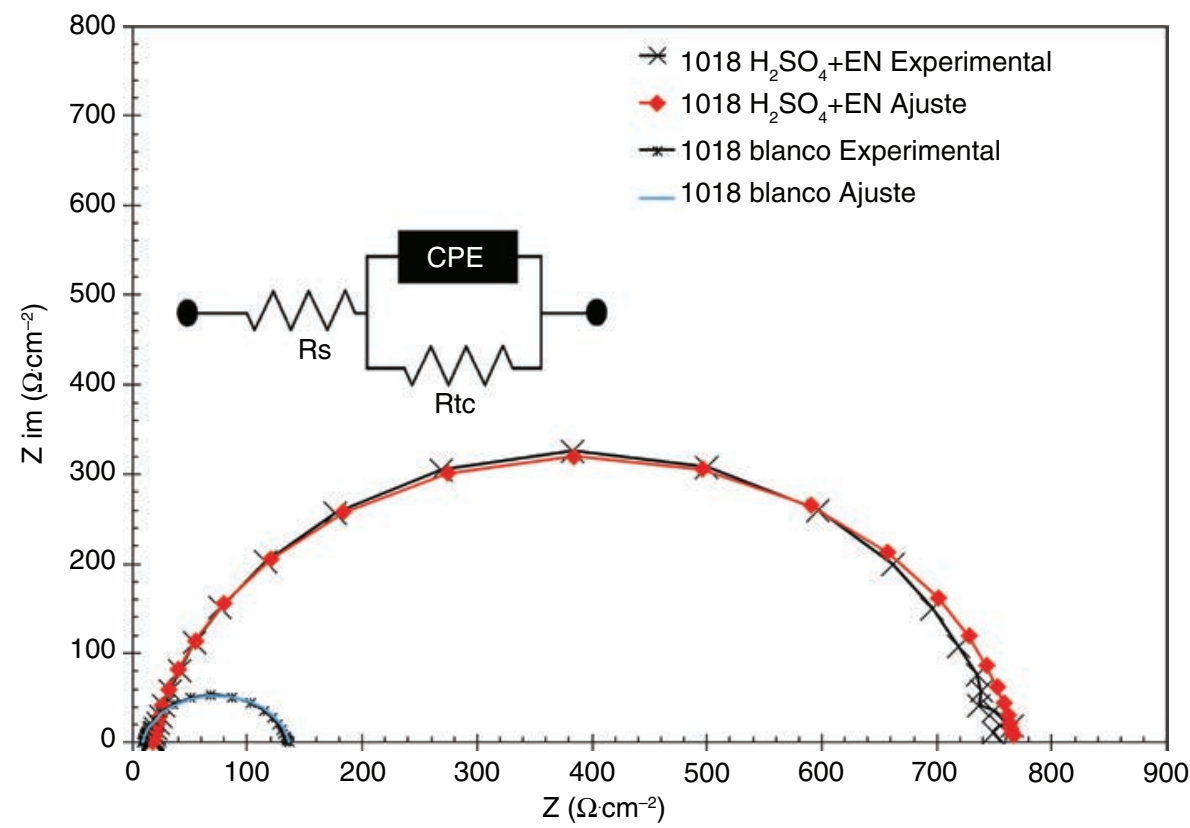

FIGURA 9. CEE seleccionado para el Ajuste de los Espectros de Impedancia para ambos casos: 1018 en $\mathrm{H}_{2} \mathrm{SO}_{4}$ y 1018 en $50 \%$ $\mathrm{H}_{2} \mathrm{SO}_{4}+50 \%$ EN.

Una forma de calcular la eficiencia del inhibidor es a través de determinar el grado de cobertura de la superficie metálica " $\theta$ " mediante la siguiente expresión (Ibrahim et al., 2011; Espinoza-Vázquez et al., 2014):

$$
\theta=\frac{\frac{1}{R_{c t}}(\text { blanco })-\frac{1}{R_{c t}}(\text { inhibidor })}{\frac{1}{R_{c t}}(\text { blanco })}
$$




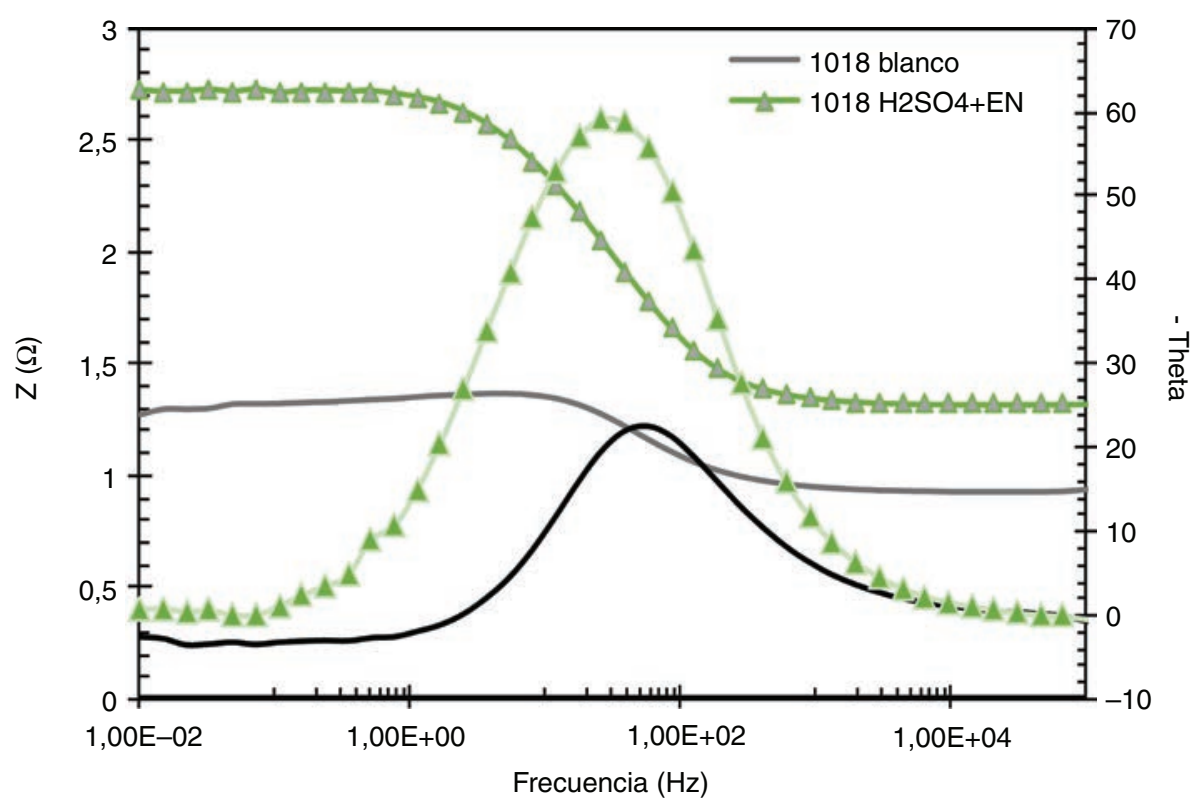

FIGURA 10. Diagrama Bode- Fase de acero 1018 en: $\mathrm{H}_{2} \mathrm{SO}_{4}$ y $50 \% \mathrm{H}_{2} \mathrm{SO}_{4}+50 \%$ EN.

TABLA 3. Resultados de $\theta^{\mathrm{a}}$ y $\mathrm{EI}^{\mathrm{b}}$ del extracto del nopal en el Acero 1018 a diferentes tiempos de exposición

\begin{tabular}{lcccc}
\hline $\mathbf{T}(\mathbf{h})$ & $\mathbf{1 / \mathbf { R } _ { \text { ct } } \text { (blanco) }}$ & $\mathbf{1 / \mathbf { R } _ { \text { ct } } \text { (inhibidor) }}$ & $\boldsymbol{\theta}$ & $\mathbf{E I} \mathbf{( \% )}$ \\
\hline 1,00 & 0,0063 & 0,0015 & 0,758 & 75,77 \\
2,50 & 0,0063 & 0,0015 & 0,758 & 75,77 \\
5,00 & 0,0057 & 0,0017 & 0,705 & 70,50 \\
7,50 & 0,0050 & 0,0015 & 0,704 & 70,40 \\
10,00 & 0,0050 & 0,0013 & 0,733 & 73,33 \\
12,50 & 0,0048 & 0,0013 & 0,723 & 72,29 \\
15,00 & 0,0047 & 0,0013 & 0,719 & 71,94 \\
17,50 & 0,0048 & 0,0013 & 0,723 & 72,32 \\
20,00 & 0,0050 & 0,0013 & 0,745 & 74,48 \\
22,50 & 0,0080 & 0,0013 & 0,843 & 84,34 \\
\hline
\end{tabular}

a. Grado de cobertura en la superficie.

b. Eficiencia de inhibición.

Donde $1 / \mathrm{R}_{\mathrm{ct}}$ es el inverso de la resistencia a la transferencia de carga cuyos valores fueron tomados de los resultados de impedancia.

La eficiencia calculada de las expresiones 4 y 5 se muestran en la Tabla 3, donde el promedio de la eficiencia del extracto del nopal como inhibidor de la corrosión es del 74,11\% en un ambiente de $\mathrm{H}_{2} \mathrm{SO}_{4}$.

$$
E I(\%)=\theta \times 100
$$

La evidencia fotografía revela que el acero 1018 inmerso en $\mathrm{H}_{2} \mathrm{SO}_{4} \quad 0,6\left(\mathrm{~mol} \cdot \mathrm{l}^{-1}\right)$ después de $24 \mathrm{~h}$ sufre un ataque uniforme acompañado de una alta densidad de picaduras profundas en toda la superficie de prueba como se muestra en las Figs. 11a y c. La severidad del ataque disminuye con la adición del extracto de nopal como se observa en las Figs. $11 \mathrm{~b}$ y d donde se identifica corrosión uniforme sin la presencia de picaduras.

\section{CONCLUSIONES}

- El extracto de nopal demostró tener propiedades de inhibición del proceso de corrosión por picaduras en $\mathrm{H}_{2} \mathrm{SO}_{4}$ desde las primeras $2,5 \mathrm{~h}$ de inmersión para el acero AISI 1018, comportándose como un inhibidor del tipo mixto durante las 24 h de exposición al medio ácido.

- Los resultados indican que se alcanzó una eficiencia de inhibición de la corrosión del $84 \%$ a las $22 \mathrm{~h}$ de inmersión, indicando que la eficiencia puede cambiar no solo por la concentración, si no con respecto al tiempo. El comportamiento de Bode ángulo demostró un cambio de la capacitancia $\mathrm{C}_{\mathrm{dl}}$ de la superficie del acero AISI 1018 con el extracto de Nopal, lo cual está ligado una mejor redistribución de las moléculas de EN sobre los sitios activos del acero, promoviendo un ataque generalizado de menor agresividad y sin la presencia de picaduras, en comparación con los daños observados en el acero expuesto únicamente al $\mathrm{H}_{2} \mathrm{SO}_{4}$ (blanco).

- Dentro de este trabajo únicamente se consideró mostrar la capacidad de inhibición frente a la corrosión que posee el EN, en un medio ácido diferente a $\mathrm{HCl}$, sin embargo, se considera necesario obtener un perfil de eficiencia 
(a)

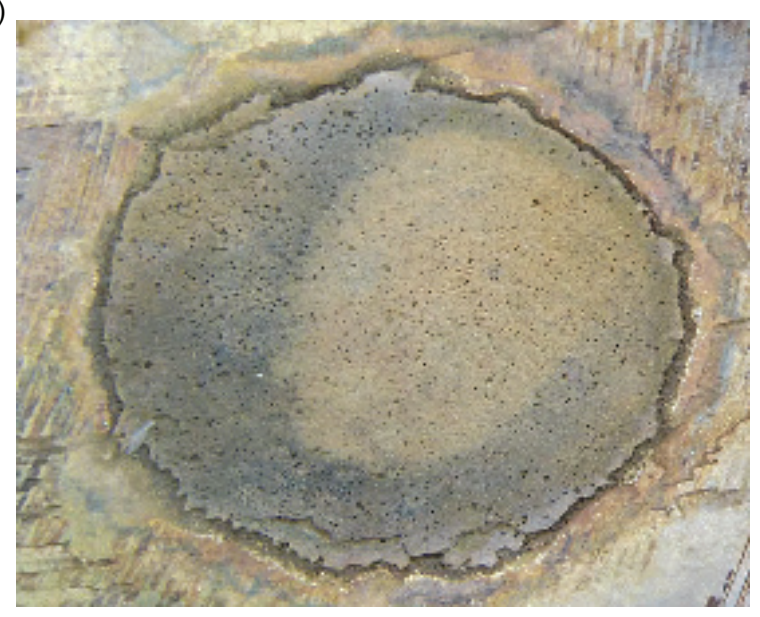

(c)

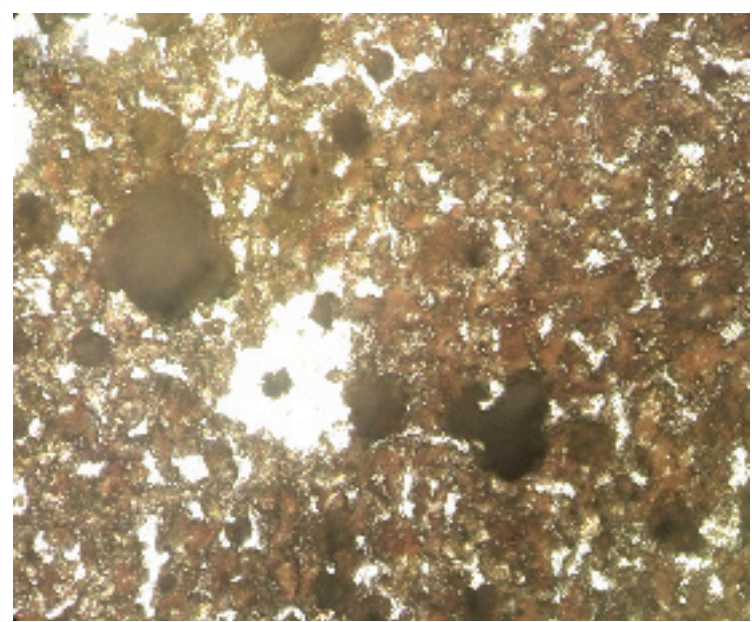

(b)

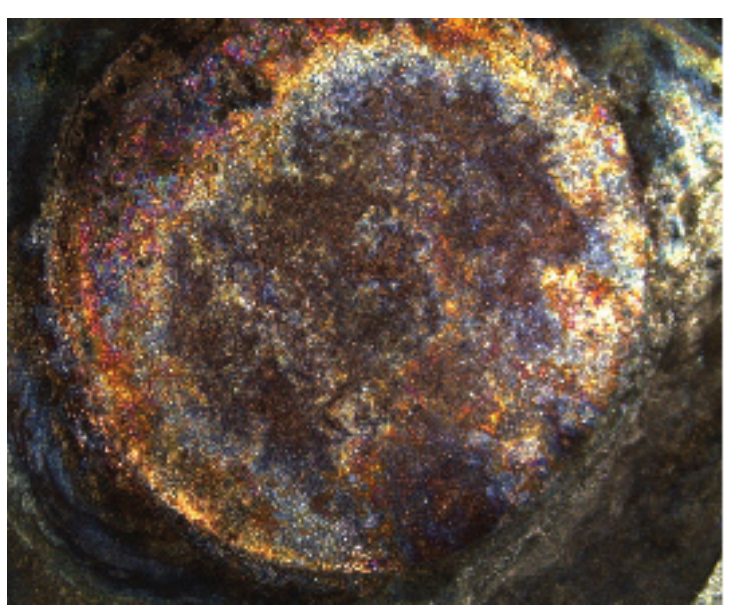

(d)

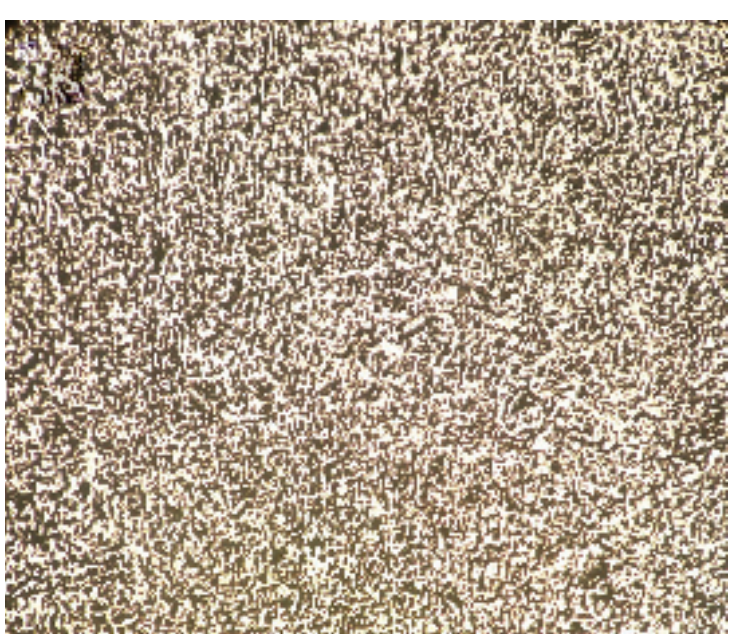

Figura 11. Micrografías obtenidas mediante el microscopio óptico a $5 \mathrm{X}$ después de las pruebas de corrosión: a y c) 1018 en $\mathrm{H}_{2} \mathrm{SO}_{4}$, b y d) 1018 en $50 \% \mathrm{H}_{2} \mathrm{SO}_{4}+50 \% \mathrm{EN}$.

a diversas concentraciones, así como a diferentes temperaturas, para estudiar el mecanismo de adsorción específico de la molécula de EN y la influencia que el compuesto del Mucílago presenta en el extracto.

\section{AGRADECIMIENTOS}

Este proyecto de investigación fue desarrollado en el Centro de Investigación y Desarrollo Tecnológico en Electroquímica S.C. (CIDETEQ) en colaboración con la Universidad Autónoma del Estado de México (UAEM), Centro Universitario UAEM Valle de México. En particular el Dr. Héctor Herrera Hernández agradece el apoyo económico otorgado a través del UAEM-SIyEA (Secretaría de Investigación y Estudios Avanzados) 3817/2014/ CID. También se agradece al estudiante Luis E. Almazán Corona por las pruebas de corrosión realizadas como parte de su residencia profesional y tesis de licenciatura. Por último, los autores reconocen al CONACyT por la aportación económica mensual por el desempeño como investigador nacional con reconocimiento SNI.

\section{REFERENCIAS}

Abreu-Quijano, M., Palomar-Pardavé, M., Cuán, A., RomeroRomo, M., Negrón-Silva, G., Álvarez-Bustamante, R., Ramírez-López, A., Herrera-Hernández, H. (2011). Quantum chemical study of 2-mercaptoimidazole, 2-Mercaptobenzimidazole, 2-Mercapto-5-Methylbenzimidazole and 2-Mercapto-5-Nitrobenzimidazole as corrosion inhibitors for steel. Int. J. Electrochem. Sci. 6 (9), 3729-3742. http:// www.electrochemsci.org/papers/vol6/6093729.pdf.

Espinoza-Vázquez, A., Negrón-Silva, G.E., GonzálezOlvera, R., Angeles-Beltrán, D., Herrera-Hernández, H., Romero-Romo, M., Palomar-Pardavé, M. (2014). Mild steel corrosion inhibition in $\mathrm{HCl}$ by di-alkyl and di-1,2,3-triazole derivatives of uracil and thymine. Mater. Chem. Phys. 145 (3), 407-417. https://doi.org/10.1016/j. matchemphys.2014.02.029.

Flores de los Ríos, J.P., Sánchez-Carrillo, M., Nava-Dino, C.G., Chacón-Nava, J.G., González-Rodríguez, J.G., HuapePadilla, E., Neri-Flores, M.A., Martínez-Villafañe, A. 
(2015). Opuntia Ficus-Indica Extract as Green Corrosion Inhibithor for Carbon Steel in 1M HClSolution. J. Spectrosc 2015, ID 714692. http://dx.doi.org/10.1155/2015/714692.

Garfias-García, E., Colin-Paniagua, F.A., Herrera-Hernández, H., Juarez-Garcia, J.M., Palomar-Padavé, M., RomeroRomo, M. (2010). Electrochemical and microscopy study of localized corrosion on a sensitized stainless steel AISI 304. ECS Transactions 29 (1), 93-102. http://dx.doi. org/10.1149/1.3532307.

Gopiraman, M., Sakunthala, P., Kesavan, D., Alexramani, V., Kim, I.S., Sulochana, N. (2011). An investigation of mild carbon steel corrosion inhibition in hydrochloric acid medium by environment friendly green inhibitors. $J$. Coat. Technol. Res. 9 (1), 15-26. http://dx.doi.org/10.1007/ s11998-011-9374-6.

Herrera-Hernández, H. Franco-Tronco, M.I., MirandaHernández, J.G., Hernández-Sánchez, E., EspinoizaVázquez, A., Fajardol, G. (2015). Gel de Aloe-Vera como Potencial Inhibidor de la Corrosión del Acero de Refuerzo Estructural. Avances en Ciencias e Ingeniería 6 (3), 9-23. http://www.redalyc.org/articulo.oa?id=323642274002.

Ibrahim, T.H., Chehade, Y., Zour, M.A. (2011). Corrosion inhibition of mild steel using potato peel extract in 2M HCL solution. Int. J. Electrochem. Sci. 6, 6542-6555. http:// www.electrochemsci.org/papers/vol6/6126542.pdf.

Ibrahim, T.H. Zour, M.A. (2011). Corrosion Inhibition of Mild Steel using Fig Leaves Extract in Hydrochloric Acid Solution. Int. J. Electrochem. Sci. 6, 6442-6455. http://www. electrochemsci.org/papers/vol6/6126442.pdf.
Ismail, M., Raja, B., Salawu, A.A. (2015). Developing Deeper Understanding of Green Inhibitors for Corrosion of Reinforcing Steel in Concrete. Chapter 7, In: Handbook of Research on Recent Development in Materials Science and Corrosion Engineering Education. Ed. Hwee, L.L. USA, pp. 118-123.

Khaled, K.F. (2010). Experimental, density function theory calculations and molecular dynamics simulations to investigate the adsorption of some thiourea derivatives on iron surface in nitric acid solutions. Appl. Surf. Sci. 256 (22), 6753-6763. http://doi.org/10.1016/j. apsusc.2010.04.085.

Palomar-Pardavé, M., Romero-Romo, M., Herrera-Hernández, H., Abreu-Quijano, M.A., Likhanova, N.V., Uruchurtu, J., Juárez-García, J.M. (2012). Influence of the alkyl chain length of 2amino 5 alkyl 1,3,4 thidiazole compounds on the corrosion inhibition of steel immersed in sulfuric acid solutions. Corros. Sci. 54, 231-243. https://doi.org/10.1016/j. corsci.2011.09.020

Sanyal, B. (1981). Organic compounds as corrosion inhibitors in different environments-A review. Prog. Org. Coat. 9 (2), 165-236. https://doi.org/10.1016/0033-0655(81)80009-X.

Singh, W.P., Bockris, J.O. (1996). Toxicity issues of organic corrosion inhibitors; applications of QSAR Model. Proceeding Corrosion 96, NACE International, Houston, USA

Torres-Acosta, A.A., Martínez-Molina, W., Alonso-Guzmán, E.M. (2012). State of the Art on Cactus Additions in AlkalineMedia as Corrosion Inhibitors. Int. J. Corr. 2012, ID 646142. http://doi.org/10.1155/2012/646142. 